\title{
Potential of DNA methylation in rectal cancer as diagnostic and prognostic biomarkers
}

\author{
Ruth Exner ${ }^{1,5}$, Walter Pulverer ${ }^{2,5}$, Martina Diem ${ }^{3}$, Lisa Spaller ${ }^{3}$, Laura Woltering ${ }^{3}$, Martin Schreiber ${ }^{4}$, \\ Brigitte Wolf ${ }^{1}$, Markus Sonntagbauer ${ }^{2}$, Fabian Schröder ${ }^{2}$, Judith Stift ${ }^{3}$, Fritz Wrba ${ }^{3}$, Michael Bergmann ${ }^{1}$, \\ Andreas Weinhäusel ${ }^{2}$ and Gerda Egger ${ }^{\star, 3}$ \\ ${ }^{1}$ Department of Surgery, Medical University of Vienna, Waehringer Guertel 18-20, A-1090 Vienna, Austria; ${ }^{2}$ AlT-Austrian Institute \\ of Technology GmbH, Health \& Environment Department, Molecular Diagnostics, Muthgasse 11/2, A-1190 Vienna, Austria; \\ ${ }^{3}$ Clinical Institute of Pathology, Medical University of Vienna, Waehringer Guertel 18-20, A-1090 Vienna, Austria and ${ }^{4}$ Department \\ of Obstetrics and Gynecology, Medical University of Vienna, Waehringer Guertel 18-20, A-1090 Vienna, Austria
}

Background: Aberrant DNA methylation is more prominent in proximal compared with distal colorectal cancers. Although a number of methylation markers were identified for colon cancer, yet few are available for rectal cancer.

\begin{abstract}
Methods: DNA methylation differences were assessed by a targeted DNA microarray for 360 marker candidates between 22 fresh frozen rectal tumour samples and 8 controls and validated by microfluidic high-throughput and methylation-sensitive qPCR in fresh frozen and formalin-fixed paraffin-embedded (FFPE) samples, respectively. The CpG island methylator phenotype (CIMP) was assessed by MethyLight in FFPE material from 78 patients with pT2 and pT3 rectal adenocarcinoma.
\end{abstract}

Results: We identified and confirmed two novel three-gene signatures in fresh frozen samples that can distinguish tumours from adjacent tissue as well as from blood with a high sensitivity and specificity of up to 1 and an AUC of 1. In addition, methylation of individual CIMP markers was associated with specific clinical parameters such as tumour stage, therapy or patients' age. Methylation of CDKN2A was a negative prognostic factor for overall survival of patients.

Conclusions: The newly defined methylation markers will be suitable for early disease detection and monitoring of rectal cancer.

Rectal cancer comprises one-third of colorectal cancers (CRCs) and is characterised by its specific anatomic localisation in the small pelvis, which makes operative resection with clear margins more difficult but allows for irradiation as an effective neoadjuvant treatment. Molecularly, distal tumours show a higher frequency of chromosomal instability (CIN) and p53 mutations but a lower frequency of microsatellite instability (MSI) and $\mathrm{CpG}$ island methylator phenotype (CIMP) compared with proximal tumours (Iacopetta, 2002). Genome-wide genetic and epigenetic analyses revealed an association of hypermutated tumours (mutation rate $>12$ per $10^{6}$ bases) with high DNA methylation levels, MSI and defects in mismatch repair-related genes as well as epigenetic MLH1 silencing (Network CGA, 2012). Generally, these hypermutated tumours were more likely to be found in the ascending colon and showed higher DNA methylation levels. Thus, molecular differences seem to follow a spatial linearity from proximal to distal locations along the colon rather than a dichotomy of proximal and distal subsites (Yamauchi et al, 2012; Bae et al, 2013).

CIMP has long been recognised in a subgroup of CRC with extraordinarily high levels of DNA methylation of $\mathrm{CpG}$ islands (Toyota et al, 1999) and has been associated with different clinical and molecular features such as patient age, gender, tumour localisation or BRAF mutation status (Weisenberger et al, 2006). In addition, a specific DNA methylation subgroup associated with KRAS mutations has been observed in CRC (Ogino et al, 2006; Shen et al, 2007; Yagi et al, 2010), which is now commonly accepted as the CIMP-low group (Hinoue et al, 2012). In-depth genome-scale analyses of DNA methylation in CRC revealed four

\footnotetext{
*Correspondence: Dr G Egger; E-mail: gerda.egger@meduniwien.ac.at
}

${ }^{5}$ These authors contributed equally to this work

Received 28 March 2015; revised 17 June 2015; accepted 30 July 2015; published online 3 September 2015 
distinct classes (CIMP-high, CIMP-low, cluster 3 and cluster 4) and suggested biological differences between the four subgroups (Hinoue et al, 2012). Intriguingly, rectal cancers were mainly associated with a distinct cluster of tumours with low cancerassociated DNA methylation.

Tumour-specific DNA methylation signatures have emerged as promising targets for biomarker development for several malignancies including CRC and might be used for early detection, prognosis and therapy response prediction in the future (Draht et al, 2012; Lange and Laird, 2013). However, discovery of markers specific for rectal cancers has been hampered by the lack of studies addressing rectal tumours as an independent entity and studies using limited numbers of candidate genes. A set of five hypermethylated tumour suppressor genes was identified in early-stage rectal cancer using a candidate gene approach and was associated with localised disease (Leong et al, 2011).

A large Asian population-based study investigating nine different bowel subsites of CRC revealed a linear association of tumour location and clinicopathological characteristics (Bae et al, 2013). In this study, CIMP was an independent poor prognostic factor for disease-free survival (DFS) and overall survival (OS) in rectal cancers but not for tumours from proximal and distal colon locations. Analysis of a set of methylated-in-tumour (MINT)specific regions revealed a two-marker panel, which was predictive of distant recurrence in early, node-negative rectal cancers and could also identify a group of patients with increased risk of local tumour recurrence, suggesting a value of this marker panel for patient stratification for neoadjuvant radiation therapy (de Maat et al, 2008, 2010). Furthermore, KRAS2 mutation and CIMP were associated with a worse 5 -year survival in a population-based study of 990 rectal cancers (Samowitz et al, 2009) and KRAS mutation and CDKN2A promoter methylation were suggested to indicate more aggressive tumours with worse prognosis (Kohonen-Corish et al, 2014). DNA methylation levels of the repetitive element LINE-1 can be used to assess genome-wide DNA methylation changes. A recent report identified LINE-1 hypomethylation in state I-II rectal cancers as a surrogate marker associated with a higher chance of tumour recurrence and decreased survival time (Benard et al, 2013).

In a recent analysis in patients with locally advanced rectal cancer CIMP positivity was associated with poor response to preoperative radiochemotherapy and significantly reduced DFS (Jo et al, 2012). MGMT and TFAP2E methylation were found predictive of response to radiochemotherapy with MGMT hypermethylation being associated with responsiveness and TFAP2E with resistance, respectively (Lange et al, 2012; Sun et al, 2013).

On the basis of these studies, we hypothesised that rectal cancers harbour aberrant tumour-specific DNA methylation, which might serve as a biomarker for clinical assessment of these cancers. We screened a set of 22 primary rectal tumours after short-time preoperative radiation therapy for their methylation status of 360 known tumour-specific methylated genes. We included eight normal peripheral blood mononuclear cell (PBMC) samples in the analyses. The rationale for using PBMCs was the identification of markers, which might be suitable for non-invasive tumour diagnostics in plasma of patients. The initial screen was followed by technical and biological validation of our findings and resulted in the discovery of a three-gene marker panel that was suitable for the classification of rectal tumour tissue. Furthermore, we evaluated the CIMP status of 78 rectal tumours isolated from formalin-fixed paraffin-embedded (FFPE) material and correlated methylation of CIMP-specific markers with clinicopathological parameters. The workflow of our analyses is depicted in Figure 1.

\section{MATERIALS AND METHODS}

Patient selection. Patients with pT2/pT3 rectal cancer, who received surgery with curative intention (R0) in the years 20002008 at the Department of Surgery at the Medical University of Vienna were included in the analyses. For the initial screen and validation fresh frozen samples $(n=22)$ were used, whereas as second larger cohort of 78 FFPE samples was used for marker confirmation and CIMP marker evaluation. Demographic data for patients from the fresh frozen screening population and the FFPECIMP population are listed in Table 1.

DNA isolation and bisulphite conversion. DNA from frozen and FFPE tissues was isolated after assessment by a pathologist. FFPE tissues were deparaffinised using xylol and rehydrated. DNA was isolated using commercially available kits according to the standard protocols (QIAamp DNA Mini Kit; Qiagen, Hilden, Germany). Bisulphite conversion was performed using commercially available kits (EpiTect; Qiagen, Hilden, Germany) according to the manufacturer's protocol.

CIMP marker testing. We employed MethyLight analyses on DNA isolated from FFPE patient samples according to the

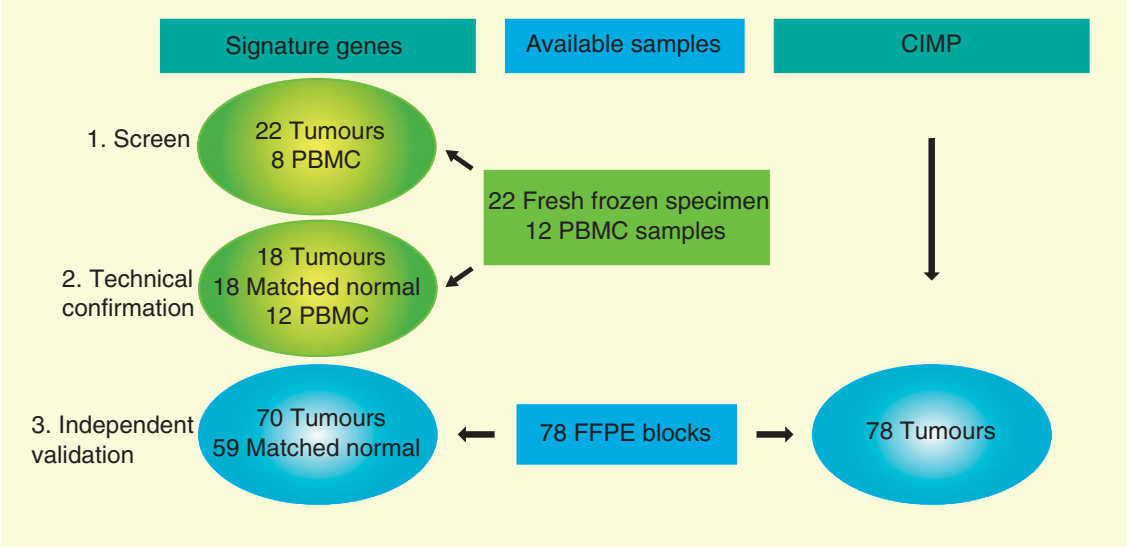

Figure 1. Workflow. Two sets of samples were available for analyses. (i) A total of 22 fresh frozen tumour specimen from patients with rectal cancer, 18 matched fresh frozen adjacent tissues and 12 PBMC samples from blood donors. These samples were used for the initial screen and for the technical confirmation of markers. (ii) A total of 78 FFPE tissue blocks from patients with rectal cancer were used for independent validation of markers and for CIMP marker analyses. 
Table 1. Demographic data of FFPE and native sample groups

\begin{tabular}{|c|c|c|c|c|}
\hline & \multicolumn{2}{|c|}{$\begin{array}{l}\text { Patiens of the FFPE } \\
\text { study group }(n=78)\end{array}$} & \multicolumn{2}{|c|}{$\begin{array}{l}\text { Patients of the native } \\
\text { study group }(n=22)\end{array}$} \\
\hline & $n$ & $\%$ & $n$ & $\%$ \\
\hline \multicolumn{5}{|l|}{ Sex } \\
\hline Male & 53 & 68 & 15 & 68 \\
\hline Female & 25 & 32 & 7 & 32 \\
\hline \multicolumn{5}{|c|}{ Age at randomisation } \\
\hline Mean & \multirow{2}{*}{\multicolumn{2}{|c|}{$\begin{array}{c}65 \\
31-86\end{array}$}} & \multirow{2}{*}{\multicolumn{2}{|c|}{$\begin{array}{c}64 \\
62-86\end{array}$}} \\
\hline Range & & & & \\
\hline \multicolumn{5}{|l|}{ Therapy } \\
\hline Short-time radiation & 59 & 76 & 22 & \\
\hline Radiochemotherapy & 8 & 10 & 0 & \\
\hline No therapy & 6 & 8 & 0 & \\
\hline ND & 5 & 6 & 0 & \\
\hline \multicolumn{5}{|l|}{ Stage } \\
\hline T2 & 33 & 42 & 0 & \\
\hline T3 & 45 & 45 & 22 & \\
\hline \multicolumn{5}{|l|}{ Node involved } \\
\hline No & 47 & 60 & 9 & 41 \\
\hline N1 & 19 & 24 & 7 & 32 \\
\hline N2 & 12 & 15 & 6 & 27 \\
\hline \multicolumn{5}{|l|}{ Dukes } \\
\hline A & 24 & 31 & 0 & \\
\hline B & 22 & 28 & 9 & 41 \\
\hline C & 28 & 36 & 13 & 59 \\
\hline D & 4 & 5 & 0 & \\
\hline \multicolumn{5}{|l|}{ Tumour location } \\
\hline Rectum & 70 & 90 & 22 & \\
\hline Rectosigmoid & 8 & 10 & & \\
\hline \multicolumn{5}{|l|}{ Recurrence } \\
\hline Local & 5 & 6 & 0 & \\
\hline Distant & 15 & 19 & 8 & 36 \\
\hline ND & 34 & 44 & 4 & 18 \\
\hline \multicolumn{5}{|l|}{ 5-year OS } \\
\hline Survived & 26 & 33 & 11 & 50 \\
\hline Not survived & 19 & 24 & 6 & 27 \\
\hline ND & 33 & 42 & 5 & 23 \\
\hline \multicolumn{5}{|l|}{ 5-year DFS } \\
\hline Recurrence & 19 & 24 & 8 & 36 \\
\hline No recurrence & 24 & 31 & 10 & 45 \\
\hline ND & 35 & 45 & 4 & 18 \\
\hline
\end{tabular}

published protocols (Campan et al, 2009) using two different CIMP panels (classical CIMP panel: CDKN2A, MINT1, MINT2, MINT31 and MLH1; new CIMP panel: CACNA1G, IGF2, NEUROG1, RUNX3 and SOCS1) (Toyota et al, 1999; Weisenberger et al, 2006). Data normalisation and percentage of methylated ratio (PMR) value calculations were done as previously described (Campan et al, 2009). A PMR that was PMR + 10 above a non-methylated control DNA was assessed as positive methylation (Weisenberger et al, 2006).

Targeted CpG-360 DNA methylation arrays. Total DNA $(600 \mathrm{ng})$ from fresh frozen tumour tissue $(n=22)$ and PBMCs ( $n=8,4$ male and 4 female) was subjected to methylation-specific restriction enzymes (MSRE) to cleave unmethylated DNA. Methylated DNA remains uncleaved and can be subjected to screening using targeted DNA microarrays for 360 methylation marker candidates (targeting $\mathrm{CpG}$ islands and human gene promoters). The main principle of the methodology was published previously (Pulverer et al, 2012). For the experiments of the present study we used the MSREs HpaII (cut site: CCGG; Fermentas, St. Leon-Rot, Germany), Hin6I (cut site: GCGC; Fermentas, St. Leon-Rot, Germany), AciI (cut site: CCGC; NEB, Frankfurt am Main, Germany) and HpyCH4IV (cut site: ACGT; NEB, Frankfurt am Main, Germany). A unit of $3 \mathrm{U}(0.3 \mu \mathrm{l})$ of each enzyme were used per digestion reaction and incubated at $37^{\circ} \mathrm{C}$ for $16 \mathrm{~h}$. The digested DNA samples were amplified in 16 multiplex PCR reactions amplifying in total 360 methylation marker candidates using biotinylated reverse primers. Pooled amplicons were detected on a targeted DNA microarray via streptavidin-Cy3 conjugate. Subsequently, significant markers were identified applying statistical tests for class comparison and class prediction. Primer sequences are available on request (Weinhäusel et al, 2009; Weinhäusel and Pulverer, 2013).

Validation of markers by MSRE-coupled qPCR. To confirm the differentially methylated gene regions from the microarray experiment DNA of tumour tissue $(n=18)$ for which adjacent normal sample material was available together with 12 PBMCs were subjected to a microfluidic high-throughput qPCR ( $\mu \mathrm{HT}-\mathrm{qPCR})$ system (Fluidigm's Biomark, San Francisco, CA, USA). Before microfluidic qPCR, a pre-amplification was carried out. For the pre-amplification the samples were digested with the four MSREs as mentioned above. The primer mixes for the pre-amplification contained 41 primer pairs at a final concentration of $200 \mathrm{~nm}$ each primer. Subsequently, qPCR was performed using EvaGreen (Biotium, Hayward, CA, USA)based detection and the following cycling conditions: $50{ }^{\circ} \mathrm{C}$ for $2 \mathrm{~min}, 95^{\circ} \mathrm{C}$ for $10 \mathrm{~min}, 45 \mathrm{cycles}$ at $95^{\circ} \mathrm{C}$ for $15 \mathrm{~s}$ and $65^{\circ} \mathrm{C}$ for $1 \mathrm{~min}$. Ct values are inversely correlated to methylation levels. In order to infer a direct association between $\mathrm{Ct}$ value and methylation status we calculated the $\Delta \mathrm{Ct}$ value (number of executed cycles $(n=45)-\mathrm{Ct}$ of sample). Primer sequences are available on request.

Validation of the identified methylation signature in FFPEderived DNA. Quantitative methylation-specific PCR (qMSP) assays were designed for the methylated allele of $5^{\prime} \mathrm{UTR}$ regions of TMEFF2, TWIST1 and PITX2 and performed in analogy to the MethyLight analyses using SYBR green detection. DNA was isolated and bisulphite converted as described above. Relative methylation was normalised to input DNA and to $100 \%$ methylated control DNA as previously described (Campan et al, 2009). Primer sequences are available on request.

Bioinformatics and statistics. For the statistical analyses of the generated array data BRB Array tools Version 4.2.1 (https:// linus.nci.nih.gov/BRB-ArrayTools.html) and R statistical software Version 2.14.2 (https://cran.r-project.org/bin/windows/base/old/ 2.14.2/) were used. To invert the indirect association between $\mathrm{Ct}$ values and methylation values, the $\Delta \mathrm{Ct}$ value was used $(45-\mathrm{Ct})$ for the calculations. Data derived from MSRE-based experiments were scale normalised and methylation differences between the sample groups were identified by comparative analyses using a random-variance $t$-test.

We used models based on different algorithms to predict the class of future samples. The models were based on the Diagonal Linear Discriminant Analysis (DLDA) (Dudoit et al, 2002), the nearest centroid method, k-nearest-neighbour classification ( $\mathrm{kNN}$ ) (Dudoit et al, 2002), support vector machines (SVM) (Ramaswamy et al, 2001) and (Bayesian) compound covariate predictor (CCP) (Radmacher et al, 2002). The DLDA is a two-class classification method based on the assumption of two Gaussian class distributions with a common covariance matrix. As a parametric model, it is highly efficient when the assumptions are met. However, the maximum likelihood estimators, which are usually plugged into the model are highly sensitive to violations from the assumptions, such as non-Gaussian distributed class distributions (e.g., outliers) or 
even when the two classes show strong differences in the covariance structure.

The nearest centroid method is an extension of the DLDA. It embeds a filter selection method by using a soft-threshold parameter to eliminate most non-contributing features. It is a fast algorithm but the choice of features is model dependent.

The $\mathrm{kNN}$ is a non-parametric multi-class classifier. It performs well when classes are heterogeneous possibly consisting of several subgroups. KNN uses the Euclidean distance to assign an instance to a class, thus it does not perform well, when there are strong (linear) dependencies between the covariates.

The SVM is non-probabilistic binary linear classifier that finds the optimal hyper plane separating the sample space into disjoint regions. The performance of the classifier depends highly on the distribution of the classes in the sample space, for example, whether it is possible to separate the classes with a linear functional. Therefore, the SVM can be extended to efficiently perform non-linear classification.

The CCP is a weighted linear combination of log-ratios. By specifying a more stringent significance level, fewer genes are included in the multivariate predictor. CCP is implicitly based on the assumption that the prior probabilities are each 0.5 . Bayesian CCP selects the differentially methylated loci for distinguishing two classes in a cross-validated training set using weighted average of the log methylation values, with the weights being the $t$-statistics of differential methylation in the training set. The values of the compound covariate scores of samples in each class in the training set are considered to have a Gaussian distribution.

The models incorporated genes that were differentially methylated between the defined groups assessed by the random-variance $t$-test. The prediction error was estimated using the leave-one-out cross-validation (LOOCV) for each model. Cross-validation error rates were estimated by repeating the LOOCV process with randomly permutated class labels. As a threshold for statistical significance $P<0.001$ and a false discovery rate $(\mathrm{FDR})<0.01$ was used for the microarray data. $P<0.05$ and FDR $<0.01$ was considered as statistically significant for the qPCR-based data. Correlation of CIMP with clinicopathological characteristics was performed with GraphPad Prism 6 software using a Spearman correlation matrix. Statistical significance was confirmed by $\chi^{2}$-test or Fisher's exact test. Kaplan-Meier statistics were used to determine the prognostic value of the CIMP marker for OS and DFS. $P$-values to Kaplan-Meier curves were calculated with log-rank tests. Multivariate analyses of the OS and DFS were performed by using a Cox proportional hazard model, unadjusted or adjusted for lymph node status and grading. Cox proportional hazard models and Kaplan-Meier plots were computed with the survival package of $\mathrm{R}$ version 2.15.1, an open-source language and environment for statistical computing (Team, 2009). We used $P<0.05$ and $95 \%$ confidence intervals for assumption of statistical significance.

Ethical and legal aspects. The project was conducted in accordance with the latest revision of the Declaration of Helsinki and the requirements of Good Clinical Practice of the European Community (CPMP/ICH/135/95). The study protocol was approved by the institutional review board ('Ethikkommission') of the Medical University of Vienna (EK-No 136/2010).

\section{RESULTS}

Identification of DNA methylation changes in cancer $v s$ control tissue. We subjected 22 fresh frozen rectal cancer DNA samples together with 8 control samples (PBMC from healthy volunteers) to microarray-based methylation analysis interrogating 360 promoters of genes known to be hypermethylated in different cancers (Weinhäusel et al, 2009). The differences in methylation intensities of the two groups (cancer $v s$ control) were identified using a random-variance $t$-test (class comparison). A total of 53 out of $360(15 \%)$ gene regions with differential methylation between the tumour tissue and the normal control samples were identified (Figure 2A). Full information for the 53 differentially methylated regions (DMRs) including gene symbol, chromosomal position, $P$-value, FDR and log fold change can be found in the Supplementary Information (Supplementary Table 1). Two-thirds $(n=35)$ of the identified DMRs showed hypermethylation in the tumour tissue. The average fold change of the microarray-derived signal intensities of the hypermethylated DMRs was $3.82 \pm 1.85$ (range, 1.2-8.35). The remaining 18 out of 53 DMRs were found hypomethylated in the tumour tissue. Their average fold change was $-1.36 \pm 0.78$ (range, -0.79 to -4.06 ). Principle component analysis also showed clustering of tumour and normal samples into two major distinct groups (Figure 2B).

Hierarchical clustering allowed for almost perfect distinction between the two sample groups (tumour vs PBMC) based on the methylation signatures of the 53 DMRs (Figure 2C). Thus, our data suggest that differential methylation of a selected number of loci might allow for distinction between rectal tumour tissue and normal PBMCs.

Identification of cancer-specific methylation signatures. Following the MSRE array experiment we chose 41 DMRs for technical replication using high-throughput MSRE qPCR using the Fluidigm Biomark platform in tumor vs PBMC DNA as well as tumour vs matched adjacent normal tissue. The DMRs contained the top 22 significant DMRs identified with the array experiment (20 hypermethylated in tumour vs PBMC with average fold change of $4.63 \pm 1.99$ : TJP2, TFPI2, two different regions for PITX2 (the two different regions target two different CpG islands of the PITX2 gene. The exact location is listed in Supplementary Table 1. The PITX2 gene has two alternative transcripts with independent promoters), SPARC, GDNF, PENK, ZNF502, RARB, CDX1, SERPINB2, SALL3, TMEFF2, BOLL, SFRP2, DCC, ESR1, SRGN, MYOD1 and WT1; and two hypomethylated DMRs with an average fold change in methylation of $-2.81 \pm 1.77$ : S100A2 and $H L A-G)$. In addition we chose 19 well-known cancer-associated genes that were included in the screen that showed high fold changes in some of the samples but did not reach statistical significance in the array experiment (SEZ6L, CHFR, CXADR, GATA4, IL1B, ZNF256, CALCA, DAPK1, CLIC4, RHOXF1, S100A8, CD24, PTGS2, MSH4, TWIST1, FMR1, NKX2-1, THBD and XIST). For qPCR confirmation we had 18 out of 22 of the initially tested fresh frozen tumour samples plus the respective adjacent tissues $(n=18+18)$ available. In addition 12 PBMC samples were included, which contained the 8 samples initially tested on the CpG-360 array. The analysis confirmed differential methylation between tumour and PBMC DNA for 15 of the 22 significant markers derived from the microarray experiments and 7 of the 19 additional candidate genes between tumour and PBMC DNA (Figure 3 and Supplementary Table 2). Using the different classification algorithms described in the 'Material and Methods' section, we were able to define a three-gene signature, comprising TFPI2, DCC and PTGS2. The combined information of these three genes allowed a perfect discrimination between tumour samples and peripheral blood with a classification success of 100\% (sensitivity: 1; specificity: 1; AUC: 1) (Figure 3 and Supplementary Table 3).

Further, we detected differential methylation of 17 out of 41 DMRs between the tumour and their adjacent normal tissue samples (Figure 4A and Supplementary Table 4). Three genes including TMEFF2, TWIST1 and PITX2 were able to classify $97-100 \%$ of the samples to the correct group dependent on the classification algorithm used (sensitivity: 0.89-1; specificity: $0.94-1$; 
A

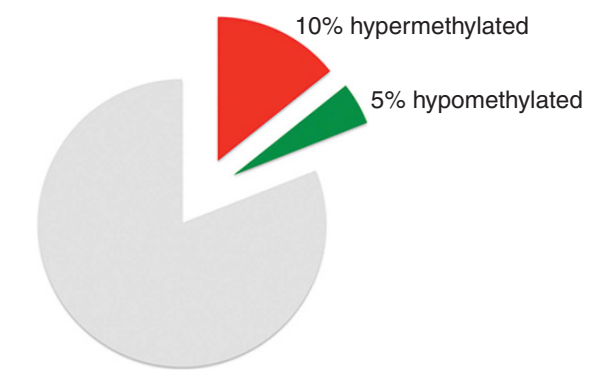

B

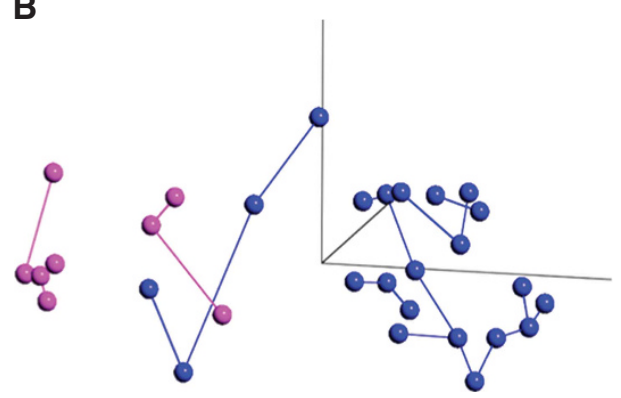

C

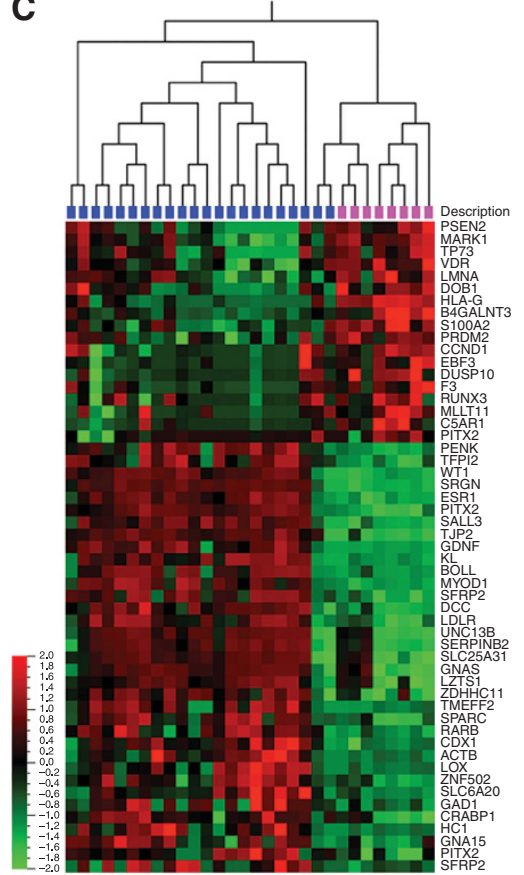

Figure 2. Microarray-based methylation screening of rectal cancers. (A) Percentage of differentially methylated gene targets identified by screening fresh frozen rectal tumours (red colour indicates regions hypermethylated in rectal cancers relative to PBMCs; green colour indicates hypomethylated regions). (B) Principal component analysis reveals separation of normal and tumour samples based on their methylation profile (purple circles represent PBMCs, blue circles represent rectal tumour samples). The two mis-clustered patients are not differing from the other patients in terms of age, gender, tumour stage/grade or preoperative therapy. The fact that these patients cluster rather with PBMCs is due to a different methylation signature of some of the genes, which might indicate that these tumours are different in terms of their molecular biology. Importantly, our identified signature gene, do classify these tumours correctly. (C) Hierarchical clustering based on the 53 top differentially methylated genes group normal and tumour samples in two distinct clusters (blue, tumour samples; purple, PBMCs; red, hypermethylated genes; green, hypomethylated genes).

AUC: 1) (Figure 4A and Supplementary Table 5). These three genes showed also promising performance to dissect the tumours from non-tumourous tissue (adjacent tissue and PBMC samples in one group) with classification values between 96 and $100 \%$ (sensitivity: 0.89-1; specificity: 0.83-1; AUC: 0.99) (Figure 4B and Supplementary Table 6).

Thus, we discovered two three-gene signatures, which were able to discriminate with high specificity and sensitivity between rectal cancer and PBMC as well as rectal cancer and adjacent normal tissue, respectively.

Confirmation of methylation signature in FFPE tissue. The three-gene signature, which allowed for a perfect discrimination between tumour tissue and matched adjacent normal tissue (TMEFF2, TWIST1 and PITX2), underwent an additional round of confirmation using DNA isolated from 70 FFPE tumour tissues and 59 FFPE adjacent normal tissues of patients with rectal cancer. All three genes showed frequent and high levels of DNA methylation in tumours relative to adjacent normal tissues with high significance, which confirmed our initial analyses in fresh frozen tumour samples (Figure 5).

CIMP analyses. In order to reassess the clinical value of CIMP for rectal cancer, we evaluated two different CIMP marker panels on DNA isolated from FFPE tumour material from patients undergoing neoadjuvant radiation therapy compared with non-radiated patients (Figure 6). We determined the CIMP status for 78 tumour samples. Application of the classical CIMP marker panel comprised of MINT1, MINT2, MINT31, CDKN2A and MLH1 revealed 15 out of $78(19.23 \%)$ as CIMP positive ( $\geqslant 3$ out of 5 markers methylated). Application of the more stringent CIMP panel comprised of CACNA1G, IGF2, NEUROG1, RUNX3 and SOCS1 identified only three CIMP-high cases ( 3 out of 5 markers methylated) within the 78 tumour samples (3.8\%). Methylation of one or two CIMP markers of this panel was detected in 26 samples (33.3\%), which might represent the previously described CIMPlow class of tumours (Hinoue et al, 2012).

To determine the associations of differential methylation of individual genes in the CIMP panels or of different CIMP classifiers with clinicopathological data we calculated a correlation matrix between the different variables using Spearman rho correlation (Figure 7A). Exposure to radiation therapy was negatively correlated with CIMP classifiers based on the classic CIMP panel and individual methylation of MLH1. Tumour stage was negatively correlated with $M L H 1$ methylation and positively correlated with MINT2 methylation. MLH1 methylation was correlated to the age of the patient at time of surgery.

To define the prognostic value of our data we computed the association of methylation status with the OS and DFS using Kaplan-Meier analyses. These analyses identified methylation of CDKN2A as a negative prognostic factor for OS of patients with rectal cancer (Figure 7B).

Next, we used Cox proportional hazards models to determine the prognostic value of $C D K N 2 A$ in univariate and multivariate analyses together with nodal status and tumour stage, which are both important prognostic clinical markers (Table 2). Our data revealed that $C D K N 2 A$ was a negative prognostic factor for OS in both univariate $(P=0.045)$ and in multivariate analysis $(P=0.017)$.

Generally, DNA methylation of selected CIMP markers correlates with clinicopathological characteristics and disease prognosis. Relating to the stringent CIMP panel (here referred to 

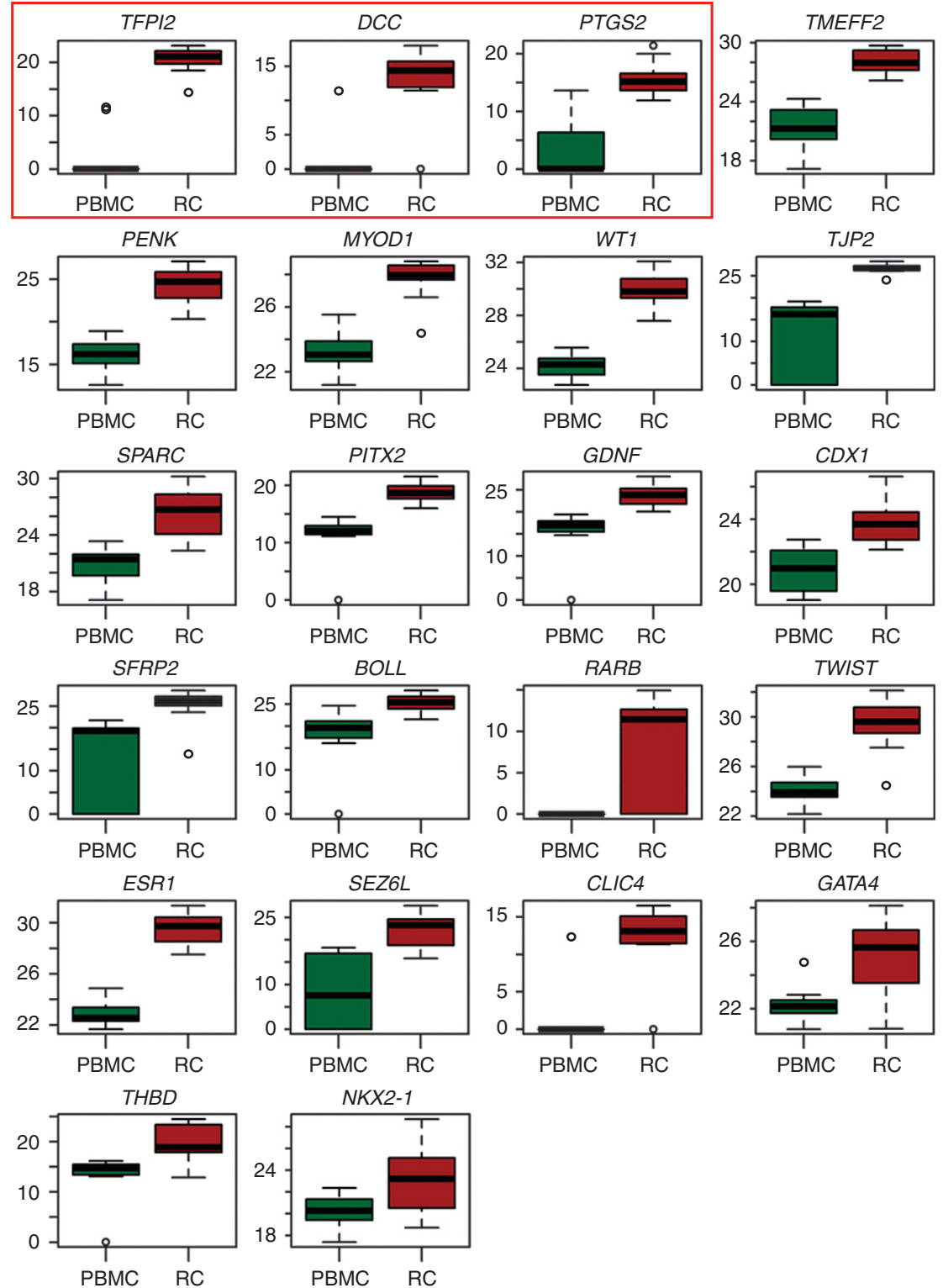

Figure 3. qPCR confirmation of a classification signature between tumour and PBMCs. Boxplots indicating the methylation differences of 22 markers, which were selected from the top differentially methylated genes on the microarray and from candidate genes, by MSRE-based qPCR. Ct values were inverted $(45-\mathrm{Ct}$ ) to reflect high and low methylation levels, respectively. TFPI2, DCC and PTGS2 (marked by a red frame) were determined as the best markers to distinguish between tumour and PBMCs by class prediction (RC, rectal carcinoma). Whiskers depict the 25 and $75 \%$ quartile of the data.

as CIMP new), our data confirm previous findings indicating that CIMP high is a rare event in rectal cancer.

\section{DISCUSSION}

Our study aimed at identifying potentially diagnostic and/or prognostic signatures of primary human rectal carcinoma based on DNA methylation. We performed a targeted DNA methylation screen interrogating 360 genes for cancer-associated marker candidates in 22 rectal tumour samples in comparison to PBMCs identifying 53 genes with differential methylation $(P<0.001)$. Validation of the 22 selected genes of the screen and 19 additional candidate markers, frequently hypermethylated in diverse cancers, analysed by class prediction identified two novel diagnostic marker sets consisting of three genes each, which were able to discriminate between tumour and PBMC as well as between tumour and adjacent tissue or normal tissue, respectively. Interestingly, the genes were not shared between the two signatures, indicating that for diagnostic applications (e.g., DNA methylation testing in cfDNA) different marker panels might be more sensitive to detect methylated tumour DNA in serum (tumour/PBMC signature) as compared with stool (tumour/adjacent normal signature).

The signature differentiating tumours from adjacent tissue and PBMCs, which we also confirmed in DNA isolated from FFPE samples using an independent technology, comprised TMEFF2 (transmembrane protein with EGF-like and two follistatin-like domains 2), TWIST1 (twist basic helix-loop-helix transcription factor1) and PITX2 (paired-like homeodomain 2). TMEFF2 methylation was first reported in bladder and colon cancer (Liang et al, 2000) and was meanwhile established as a marker for non-invasive testing of serum, urine or stool for different cancer entities (Lee et al, 2012; Elliott et al, 2013; Monteiro-Reis 

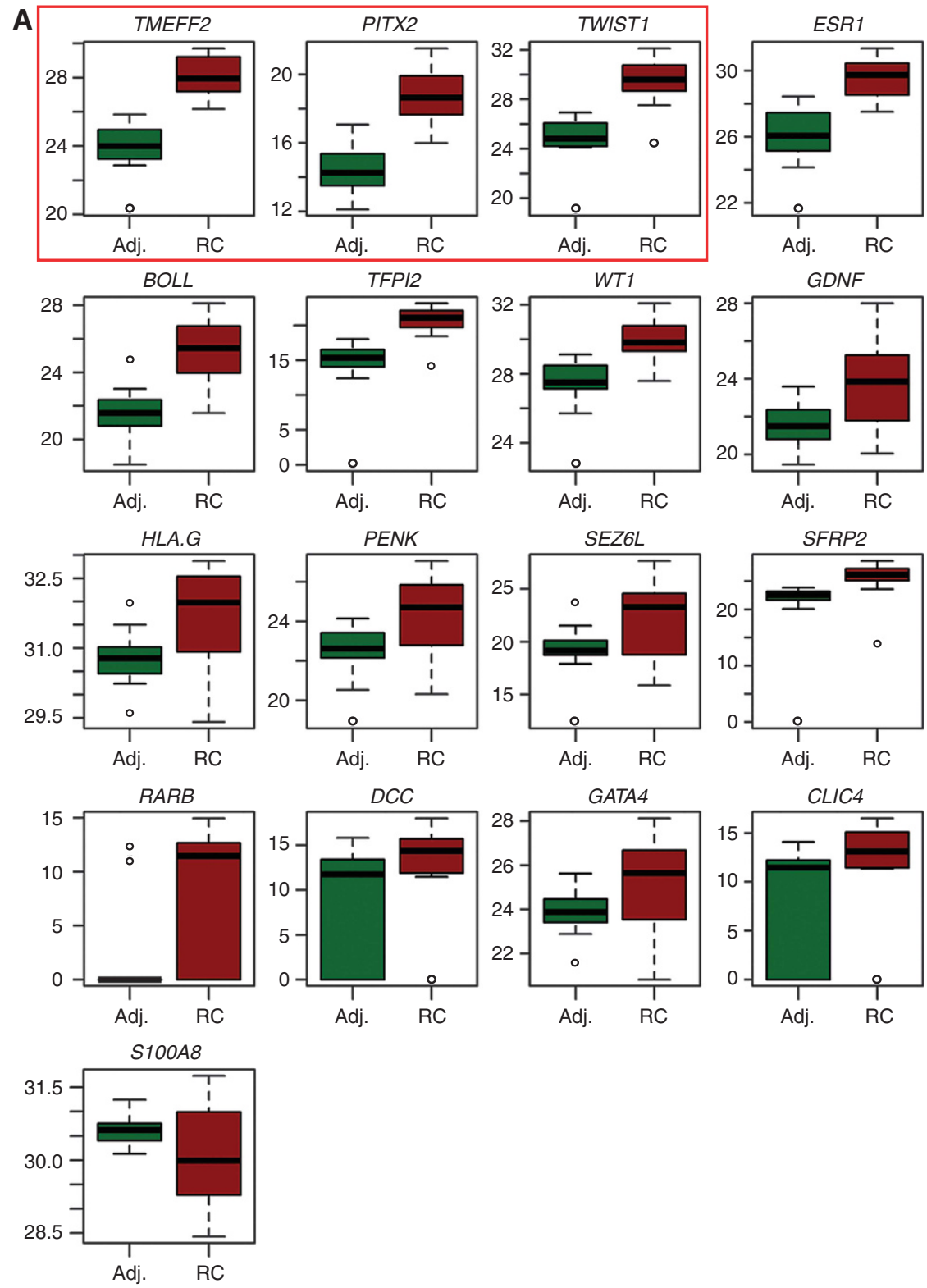

B
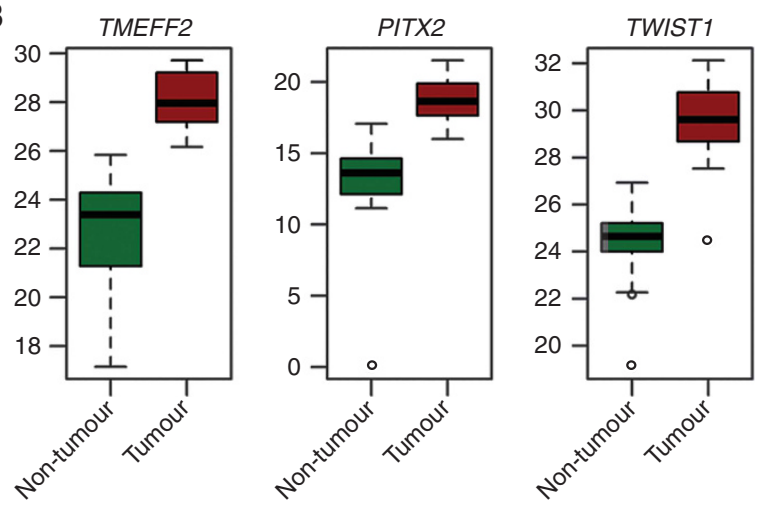

Figure 4. qPCR confirmation of a classification signature between normal and tumour. (A) Boxplots indicating the 17 markers, which were significantly differentially methylated between normal adjacent and tumour tissue as determined by MSRE-coupled qPCR. Ct values are depicted as $(45-\mathrm{ct}$ ) to reflect the methylation levels, directly (adj., adjacent normal; RC, rectal carcinoma). TMEF2, PITX2 and TWIST (marked by a red frame) were determined as the best markers to distinguish normal adjacent from tumour tissue by the classification algorithm. (B) Boxplots showing classification signature genes that best distinguish non-tumour (adjacent normal and PBMC) from tumour tissues as determined by class prediction. Whiskers depict the 25 and $75 \%$ quartile of the data. 


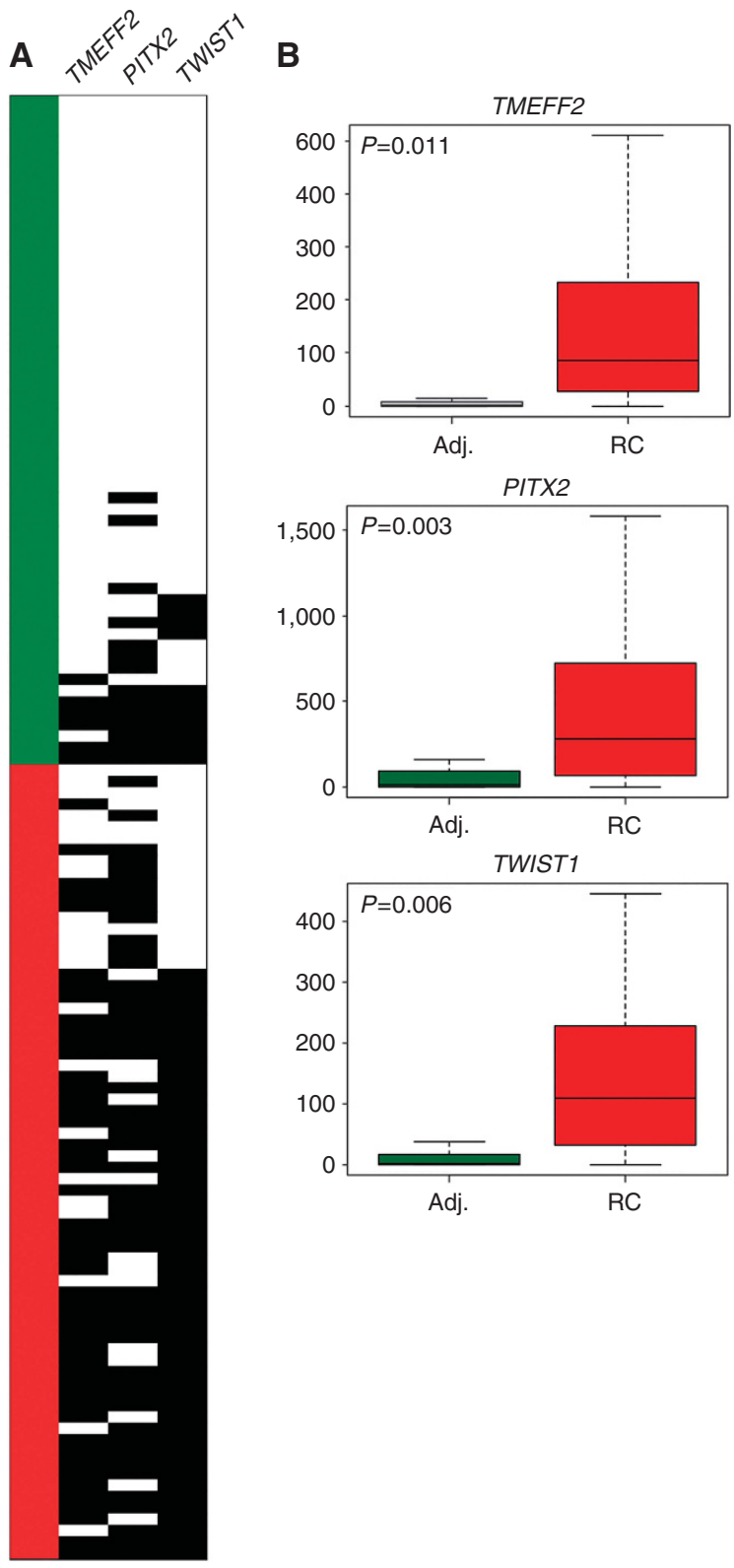

Figure 5. Confirmation of classification signature in a FFPE sample cohort. (A) Dichotomous heatmap indicating methylation frequency of TMEF2, PITX2 and TWIST (columns) in normal adjacent (green) and tumour (red) samples (rows) isolated from FFPE material using ms-qPCR (black, methylated; white, unmethylated). Samples were defined as methylated if their PMR value was $>$ PMR of an unmethylated control $D N A+10$. (B) Boxplots indicating relative methylation of tumour and adjacent normal samples analysed as in $\mathbf{A}$. Significance was calculated using unpaired $t$-tests. Whiskers depict the 25 and $75 \%$ quartile of the data.

et al, 2013). The transcription factors TWIST1 and PITX2 are both hypermethylated in different cancers and were suggested as potential biomarkers. Especially PITX2 methylation has been well established as a prognostic biomarker for breast and prostate cancer (Mikeska et al, 2012). Together, our data suggest that genes commonly methylated in cancers might be useful as markers for rectal cancer diagnostics and it will be interesting to study their prognostic and/or predictive value in a larger cohort in the future.

The signature differentiating tumours from PBMCs contained PTGS2 (prostaglandin-endoperoxide synthase 2), DCC (deleted in

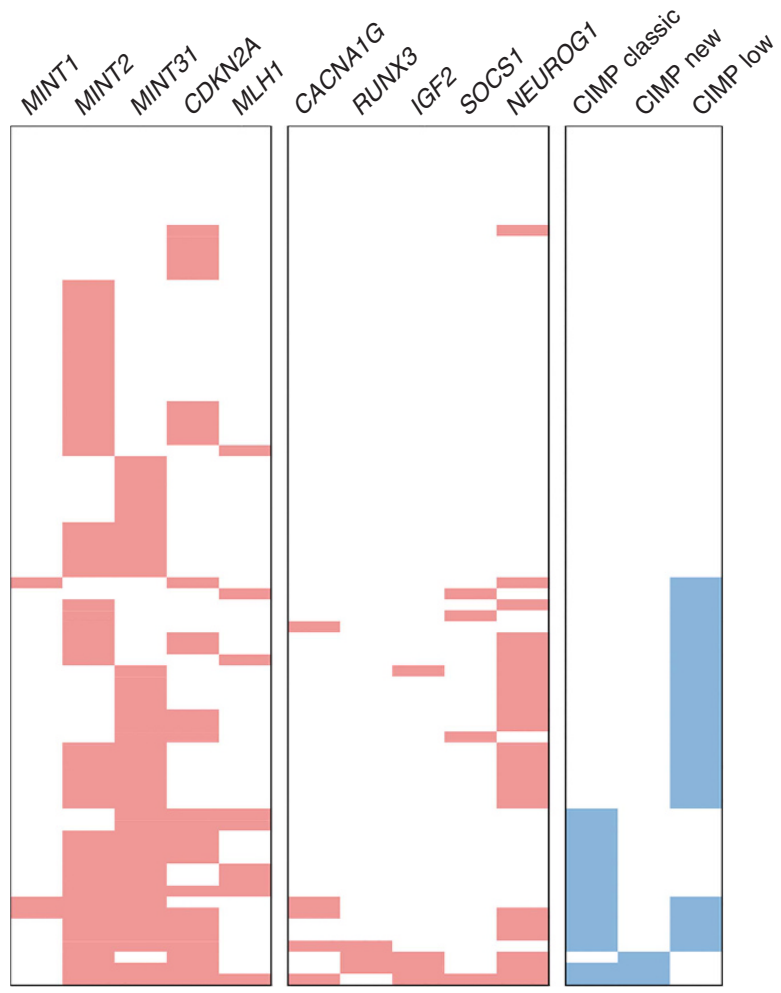

Figure 6. CIMP analysis of rectal tumours from a FFPE sample cohort. Methylation of indicated CIMP markers was performed in 78 rectal tumour samples using MethyLight. The dichotomous heatmap on the left indicates methylated (red) or unmethylated (white) markers in each sample (rows). Methylated regions were integrated into different CIMP panels shown in the histograms on the right (blue) including: CIMP classic ( 3 out of 5 of the classic makers MINT1, MINT2, MINT31, CDKN2A and MLH1 methylated); CIMP new (3 out of 5 or the new CIMP markers CACNA1G, RUNX3, IGF2, SOCS1 and NEUROG1 methylated); and CIMP low (one or two markers of the new panel methylated).

colorectal carcinoma) and TFPI2 (tissue factor pathway inhibitor 2). PTGS2 also known as COX-2 (cyclooxygenase-2) is the ratelimiting enzyme in the prostaglandin biosynthesis. Although overexpression of COX-2 has been reported for gastrointestinal tumours and COX inhibitors are promising antitumour drugs (Jendrossek, 2013), methylation of COX-2 was detected in $13 \%$ of sporadic colorectal cancers and has been associated with CIMPpositive tumours (Toyota et al, 2000). Our data imply that COX2 methylation might also be a marker for rectal cancers, suggesting that COX inhibitors might not be efficacious in rectal tumours.

$D C C$ is a tumour suppressor and both deletion and promoter methylation of $D C C$ are involved in its frequent downregulation in colorectal cancer (Tanaka et al, 1991; Derks et al, 2009).

TFPI2 belongs to a family of Kunitz-type serine protease inhibitors, implicated in tissue remodelling and proliferation. TFPI2 was suggested to act as a tumour suppressor and it was found methylated in different tumour types including pancreatic, cervical, gastric or prostate carcinoma (Matsubayashi et al, 2006; Sova et al, 2006; Jee et al, 2009; Ribarska et al, 2010). Furthermore, TFPI2 methylation was identified as a specific and sensitive marker for CRC detection in stool and serum (Glockner et al, 2009; Hibi et al, 2011).

Regarding CIMP occurrence, we identified only $3.8 \%$ of tumour samples taken from a retrospective cohort of 78 rectal cancer patients as CIMP positive according to the described marker panel comprised of CACNA1G, IGF2, NEUROG1, RUNX3 and SOCS1 

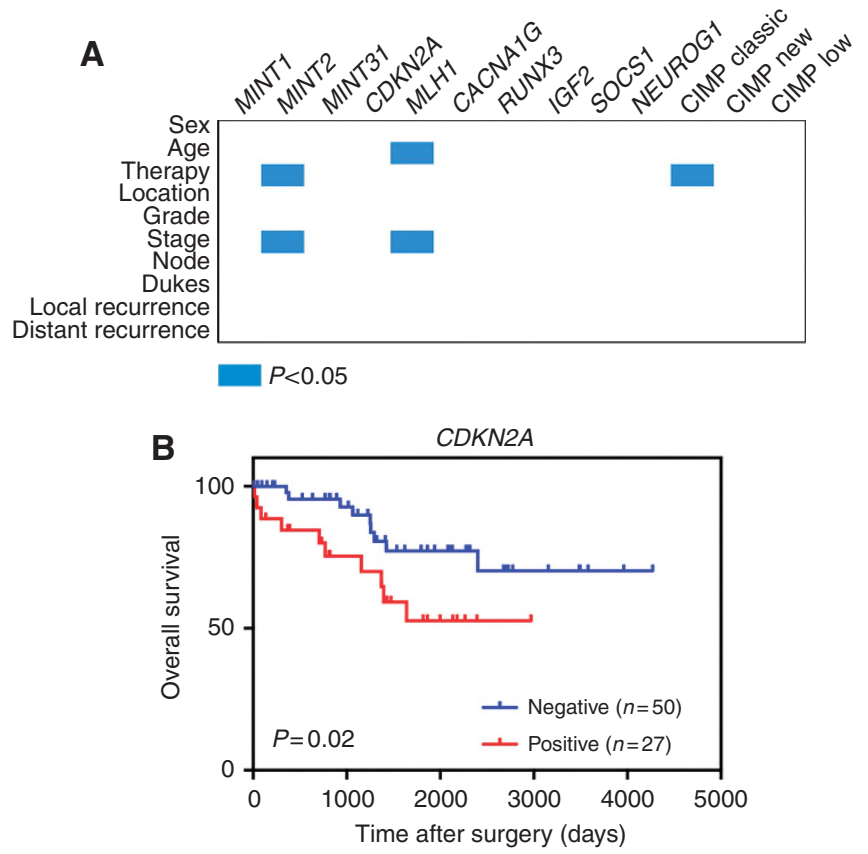

Figure 7. Correlation of methylation data with clinicopathological characteristics. (A) Spearman rho correlation matrix of methylation data (horizontal) and clinical variables (vertical). Blue coloured squares indicate significant correlations. (B) Kaplan-Meier survival statistics showing significant association between methylation of CDKN2A and OS of patients (negative, unmethylated CDKN2A; positive, methylated CDKN2A).

Table 2. Univariable and multivariable analyses of the overall survival using a Cox proportional hazards model

\begin{tabular}{|c|c|c|c|c|c|}
\hline & & Unive & riable & Multivaria & ble \\
\hline Variable & Subcategory & HR & $\begin{array}{c}P \text { - } \\
\text { value }\end{array}$ & Covariables & $\begin{array}{c}P- \\
\text { value }\end{array}$ \\
\hline CDKN2A & Pos $=0, \mathrm{Neg}=1$ & 2.54 & 0.045 & & 0.017 \\
\hline Nodal status & $\begin{array}{l}\mathrm{pNO}=0 \\
\mathrm{pN}+=1\end{array}$ & 2.28 & 0.074 & $\begin{array}{l}\text { Treated as } \\
\text { covariable } 1\end{array}$ & \\
\hline Stage & $\mathrm{pT} 2=2, \mathrm{pT} 3=3$ & 4.48 & 0.052 & $\begin{array}{l}\text { Treated as } \\
\text { covariable } 2\end{array}$ & \\
\hline
\end{tabular}

(Weisenberger et al, 2006). This corresponds well to previous data describing CIMP as being rare in rectal cancer and increasing linearly from the rectum to the ascending colon (Jo et al, 2012; Yamauchi et al, 2012). However, application of the classic CIMP marker panel including CDKN2A, MINT1, MINT2, MINT31 and $M L H 1$ revealed a positivity of $19.23 \%$. Importantly, CDKN2A methylation was of prognostic relevance in Kaplan-Meier and Cox regression analyses.

The clinical impact of CIMP in rectal cancer has recently been investigated and poor DFS was found in patients with CIMPpositive rectal cancers (Jo et al, 2012) and colorectal cancers (Barault et al, 2008; Dahlin et al, 2010). KRAS mutation and CDKN2A methylation was found to have an adverse effect on survival and predicts recurrence of rectal cancer in a recent study investigating a cohort of 381 rectal cancers (Kohonen-Corish et al, 2014). In contrast to previous studies, we included mainly patients undergoing neoadjuvant radiation therapy or radiochemotherapy in our sample collective. We believe that this might be important, as available tissue for the biomarker assessment of rectal cancer might frequently be only available after such neoadjuvant treatment. Intriguingly, we detected an association of radiation therapy and loss of methylation in the classic CIMP markers. This might imply that radiation therapy interferes with DNA methylation directly or that CIMP-positive cells are more sensitive to radiation therapy and thus selected for in the heterogeneous tumour. There is controversial data relating to alterations of DNA methylation after irradiation of cancer cells (Kim et al, 2013). In vitro experiments measuring DNA methylation after radiation of MCF7 breast cancer cells observed no significant overall changes of methylation but a delayed hypomethylation of candidate genes identified by a methylation screen $\sim 2$ weeks after exposure to radiation (Kuhmann et al, 2011). A recent genome-wide methylation study of breast cancer tissue of patients before and after radiation therapy revealed a dose-dependent change in DNA methylation in a set of genes involving both hyper- and hypomethylation (Halvorsen et al, 2014). In addition the authors found that methylation of a panel of five genes before radiation was associated with response to radiation therapy. Moreover, a link between DNA methylation and radiation therapy is evident from studies suggesting that pretreatment and DNA hypomethylation of colon cancer cells amongst others with DNA methyltransferase inhibitors can radiosensitise tumour cells (Cho et al, 2009; De Schutter and Nuyts, 2009; Hofstetter et al, 2010). Furthermore, methylation of genes implicated in DNA repair mechanisms might be surrogates for radiation sensitivity. For example, methylation or loss of $M L H 1$, which is implicated in DNA mismatch repair during DNA replication and interacts with apoptotic pathways, was suggested to predict an improved response to radiation (Shin et al, 2013). However, other reports suggested that MSI could not predict response to neoadjuvant radiotherapy in advanced rectal cancer (Du et al, 2013) or that MLH1-positive tumours show a higher response rate to neoadjuvant chemoradiotherapy (Bertolini et al, 2007). Our study included only a limited number of nonirradiated tumours, which might have biased our results. Thus, it will be instrumental to confirm our findings in a larger set of tumours and to further investigate the correlation of mismatch repair deficiency and radiosensitivity in more detail.

In summary, our data underline the relevance of DNA methylation in rectal tumours. The identification of a new set of methylation markers now provides the basis to apply and investigate the potential of these for diagnosis and prognosis for rectal carcinoma.

\section{ACKNOWLEDGEMENTS}

GE was funded by the Austrian Science Foundation (FWF) (P 27616 and V 102). Selected analyses were performed using BRBArrayTools developed by Dr Richard Simon and BRB-ArrayTools Development Team. We are thankful to Tobias Reinstadler for technical support and conducting qPCR analyses on FFPE samples.

\section{CONFLICT OF INTEREST}

The authors declare no conflict of interest.

\section{REFERENCES}

Bae JM, Kim JH, Cho NY, Kim TY, Kang GH (2013) Prognostic implication of the CpG island methylator phenotype in colorectal cancers depends on tumour location. Br J Cancer 109(4): 1004-1012.

Barault L, Charon-Barra C, Jooste V, de la Vega MF, Martin L, Roignot P, Rat P, Bouvier AM, Laurent-Puig P, Faivre J, Chapusot C, Piard F (2008) Hypermethylator phenotype in sporadic colon cancer: study on a population-based series of 582 cases. Cancer Res 68(20): 8541-8546. 
Benard A, van de Velde CJ, Lessard L, Putter H, Takeshima L, Kuppen PJ, Hoon DS (2013) Epigenetic status of LINE-1 predicts clinical outcome in early-stage rectal cancer. Br J Cancer 109(12): 3073-3083.

Bertolini F, Bengala C, Losi L, Pagano M, Iachetta F, Dealis C, Jovic G, Depenni R, Zironi S, Falchi AM, Luppi G, Conte PF (2007) Prognostic and predictive value of baseline and posttreatment molecular marker expression in locally advanced rectal cancer treated with neoadjuvant chemoradiotherapy. Int J Radiat Oncol Biol Phys 68(5): 1455-1461.

Campan M, Weisenberger DJ, Trinh B, Laird PW (2009) MethyLight. Methods Mol Biol 507: 325-337.

Cho HJ, Kim SY, Kim KH, Kang WK, Kim JI, Oh ST, Kim JS, An CH (2009) The combination effect of sodium butyrate and 5-Aza-2'-deoxycytidine on radiosensitivity in RKO colorectal cancer and MCF-7 breast cancer cell lines. World J Surg Oncol 7: 49.

Dahlin AM, Palmqvist R, Henriksson ML, Jacobsson M, Eklof V, Rutegard J, Oberg A, Van Guelpen BR (2010) The role of the CpG island methylator phenotype in colorectal cancer prognosis depends on microsatellite instability screening status. Clin Cancer Res 16(6): 1845-1855.

de Maat MF, van de Velde CJ, Benard A, Putter H, Morreau H, van Krieken JH, Meershoek Klein-Kranenbarg E, de Graaf EJ, Tollenaar RA, Hoon DS (2010) Identification of a quantitative MINT locus methylation profile predicting local regional recurrence of rectal cancer. Clin Cancer Res 16(10): 2811-2818.

de Maat MF, van de Velde CJ, van der Werff MP, Putter H, Umetani N, Klein-Kranenbarg EM, Turner RR, van Krieken JH, Bilchik A, Tollenaar RA, Hoon DS (2008) Quantitative analysis of methylation of genomic loci in early-stage rectal cancer predicts distant recurrence. J Clin Oncol 26(14): 2327-2335.

De Schutter H, Nuyts S (2009) Radiosensitizing potential of epigenetic anticancer drugs. Anticancer Agents Med Chem 9(1): 99-108.

Derks S, Bosch LJ, Niessen HE, Moerkerk PT, van den Bosch SM, Carvalho B, Mongera S, Voncken JW, Meijer GA, de Bruine AP, Herman JG, van Engeland M (2009) Promoter CpG island hypermethylation- and H3K9me3 and H3K27me3-mediated epigenetic silencing targets the deleted in colon cancer (DCC) gene in colorectal carcinogenesis without affecting neighboring genes on chromosomal region 18q21. Carcinogenesis 30(6): 1041-1048.

Draht MX, Riedl RR, Niessen H, Carvalho B, Meijer GA, Herman JG, van Engeland M, Melotte V, Smits KM (2012) Promoter CpG island methylation markers in colorectal cancer: the road ahead. Epigenomics 4(2): 179-194.

Du C, Zhao J, Xue W, Dou F, Gu J (2013) Prognostic value of microsatellite instability in sporadic locally advanced rectal cancer following neoadjuvant radiotherapy. Histopathology 62(5): 723-730.

Dudoit S, Fridlyand J, Speed TP (2002) Comparison of discrimination methods for the classification of tumors using gene expression data. J Am Stat Assoc 97(457): 77-87.

Elliott GO, Johnson IT, Scarll J, Dainty J, Williams EA, Garg D, Coupe A, Bradburn DM, Mathers JC, Belshaw NJ (2013) Quantitative profiling of $\mathrm{CpG}$ island methylation in human stool for colorectal cancer detection. Int J Colorectal Dis 28(1): 35-42.

Glockner SC, Dhir M, Yi JM, McGarvey KE, Van Neste L, Louwagie J, Chan TA, Kleeberger W, de Bruine AP, Smits KM, Khalid-de Bakker CA, Jonkers DM, Stockbrugger RW, Meijer GA, Oort FA, Iacobuzio-Donahue C, Bierau K, Herman JG, Baylin SB, Van Engeland M, Schuebel KE, Ahuja N (2009) Methylation of TFPI2 in stool DNA: a potential novel biomarker for the detection of colorectal cancer. Cancer Res 69(11): 4691-4699.

Halvorsen AR, Helland A, Fleischer T, Haug KM, Grenaker Alnaes GI, Nebdal D, Syljuasen RG, Touleimat N, Busato F, Tost J, Saetersdal AB, BorresenDale AL, Kristensen V, Edvardsen H (2014) Differential DNA methylation analysis of breast cancer reveals the impact of immune signaling in radiation therapy. Int J Cancer 135(9): 2085-2095.

Hibi K, Goto T, Shirahata A, Saito M, Kigawa G, Nemoto H, Sanada Y (2011) Detection of TFPI2 methylation in the serum of colorectal cancer patients. Cancer Lett 311(1): 96-100.

Hinoue T, Weisenberger DJ, Lange CP, Shen H, Byun HM, Van Den Berg D, Malik S, Pan F, Noushmehr H, van Dijk CM, Tollenaar RA, Laird PW (2012) Genome-scale analysis of aberrant DNA methylation in colorectal cancer. Genome Res 22(2): 271-282.

Hofstetter B, Niemierko A, Forrer C, Benhattar J, Albertini V, Pruschy M, Bosman FT, Catapano CV, Ciernik IF (2010) Impact of genomic methylation on radiation sensitivity of colorectal carcinoma. Int J Radiat Oncol Biol Phys 76(5): 1512-1519.
Iacopetta B (2002) Are there two sides to colorectal cancer? Int J Cancer 101(5): 403-408.

Jee CD, Kim MA, Jung EJ, Kim J, Kim WH (2009) Identification of genes epigenetically silenced by CpG methylation in human gastric carcinoma. Eur J Cancer 45(7): 1282-1293.

Jendrossek V (2013) Targeting apoptosis pathways by Celecoxib in cancer. Cancer Lett 332(2): 313-324.

Jo P, Jung K, Grade M, Conradi LC, Wolff HA, Kitz J, Becker H, Ruschoff J, Hartmann A, Beissbarth T, Muller-Dornieden A, Ghadimi M, SchneiderStock R, Gaedcke J (2012) CpG island methylator phenotype infers a poor disease-free survival in locally advanced rectal cancer. Surgery 151(4): 564-570.

Kim JG, Park MT, Heo K, Yang KM, Yi JM (2013) Epigenetics meets radiation biology as a new approach in cancer treatment. Int J Mol Sci 14(7): 15059-15073.

Kohonen-Corish MR, Tseung J, Chan C, Currey N, Dent OF, Clarke S, Bokey L, Chapuis PH (2014) KRAS mutations and CDKN2A promoter methylation show an interactive adverse effect on survival and predict recurrence of rectal cancer. Int J Cancer 134(12): 2820-2828.

Kuhmann C, Weichenhan D, Rehli M, Plass C, Schmezer P, Popanda O (2011) DNA methylation changes in cells regrowing after fractioned ionizing radiation. Radiother Oncol 101(1): 116-121.

Lange CP, Campan M, Hinoue T, Schmitz RF, van der Meulen-de Jong AE, Slingerland H, Kok PJ, van Dijk CM, Weisenberger DJ, Shen H, Tollenaar RA, Laird PW (2012) Genome-scale discovery of DNA-methylation biomarkers for blood-based detection of colorectal cancer. PLoS One 7(11): e50266.

Lange CP, Laird PW (2013) Clinical applications of DNA methylation biomarkers in colorectal cancer. Epigenomics 5(2): 105-108.

Lee SM, Park JY, Kim DS (2012) Methylation of TMEFF2 gene in tissue and serum DNA from patients with non-small cell lung cancer. Mol Cells 34(2): 171-176

Leong KJ, Wei W, Tannahill LA, Caldwell GM, Jones CE, Morton DG, Matthews GM, Bach SP (2011) Methylation profiling of rectal cancer identifies novel markers of early-stage disease. Br J Surg 98(5): 724-734.

Liang G, Robertson KD, Talmadge C, Sumegi J, Jones PA (2000) The gene for a novel transmembrane protein containing epidermal growth factor and follistatin domains is frequently hypermethylated in human tumor cells. Cancer Res 60(17): 4907-4912.

Matsubayashi H, Canto M, Sato N, Klein A, Abe T, Yamashita K, Yeo CJ, Kalloo A, Hruban R, Goggins M (2006) DNA methylation alterations in the pancreatic juice of patients with suspected pancreatic disease. Cancer Res 66(2): 1208-1217.

Mikeska T, Bock C, Do H, Dobrovic A (2012) DNA methylation biomarkers in cancer: progress towards clinical implementation. Expert Rev Mol Diagn 12(5): 473-487.

Monteiro-Reis S, Leca L, Almeida M, Antunes L, Monteiro P, Dias PC, Morais A, Oliveira J, Henrique R, Jeronimo C (2013) Accurate detection of upper tract urothelial carcinoma in tissue and urine by means of quantitative GDF15, TMEFF2 and VIM promoter methylation. Eur J Cancer 50(1): 226-233.

Network CGA (2012) Comprehensive molecular characterization of human colon and rectal cancer. Nature 487(7407): 330-337.

Ogino S, Kawasaki T, Kirkner GJ, Loda M, Fuchs CS (2006) CpG island methylator phenotype-low (CIMP-low) in colorectal cancer: possible associations with male sex and KRAS mutations. J Mol Diagn 8(5): $582-588$.

Pulverer W, Wielscher M, Panzer-Grumayer R, Plessl T, Kriegner A, Vierlinger K, Weinhausel A (2012) The stem cell signature of CHH/CHG methylation is not present in 271 cancer associated $5^{\prime}$ UTR gene regions. Biochimie 94(11): 2345-2352.

Radmacher MD, McShane LM, Simon R (2002) A paradigm for class prediction using gene expression profiles. J Comput Biol 9(3): 505-511.

Ramaswamy S, Tamayo P, Rifkin R, Mukherjee S, Yeang CH, Angelo M, Ladd C, Reich M, Latulippe E, Mesirov JP, Poggio T, Gerald W, Loda M, Lander ES, Golub TR (2001) Multiclass cancer diagnosis using tumor gene expression signatures. Proc Natl Acad Sci USA 98(26): 15149-15154.

Ribarska T, Ingenwerth M, Goering W, Engers R, Schulz WA (2010) Epigenetic inactivation of the placentally imprinted tumor suppressor gene TFPI2 in prostate carcinoma. Cancer Genomics Proteomics 7(2): $51-60$. 
Samowitz WS, Curtin K, Wolff RK, Tripp SR, Caan BJ, Slattery ML (2009) Microsatellite instability and survival in rectal cancer. Cancer Causes Control 20(9): 1763-1768.

Shen L, Toyota M, Kondo Y, Lin E, Zhang L, Guo Y, Hernandez NS, Chen X, Ahmed S, Konishi K, Hamilton SR, Issa JP (2007) Integrated genetic and epigenetic analysis identifies three different subclasses of colon cancer. Proc Natl Acad Sci USA 104(47): 18654-18659.

Shin JS, Tut TG, Yang T, Lee CS (2013) Radiotherapy response in microsatellite instability related rectal cancer. Korean J Pathol 47(1): 1-8.

Sova P, Feng Q, Geiss G, Wood T, Strauss R, Rudolf V, Lieber A, Kiviat N (2006) Discovery of novel methylation biomarkers in cervical carcinoma by global demethylation and microarray analysis. Cancer Epidemiol Biomarkers Prev 15(1): 114-123.

Sun W, Sun Y, Zhu M, Wang Z, Zhang H, Xin Y, Jiang G, Guo X, Zhang Z, Liu Y (2013) The role of plasma cell-free DNA detection in predicting preoperative chemoradiotherapy response in rectal cancer patients. Oncol Rep 31(3): 1466-1472.

Tanaka K, Oshimura M, Kikuchi R, Seki M, Hayashi T, Miyaki M (1991) Suppression of tumorigenicity in human colon carcinoma cells by introduction of normal chromosome 5 or 18. Nature 349(6307): $340-342$.

Team RDC (2009) A Language and Environment for Statistical Computing. R Foundation for Statistical Computing: Vienna, Austria.

Toyota M, Ahuja N, Ohe-Toyota M, Herman JG, Baylin SB, Issa JP (1999) $\mathrm{CpG}$ island methylator phenotype in colorectal cancer. Proc Natl Acad Sci USA 96(15): 8681-8686.

Toyota M, Shen L, Ohe-Toyota M, Hamilton SR, Sinicrope FA, Issa JP (2000) Aberrant methylation of the Cyclooxygenase $2 \mathrm{CpG}$ island in colorectal tumors. Cancer Res 60(15): 4044-4048.

Weinhäusel A, Pulverer W (2013) DNA methylation biomarkers for identification of colorectal cancer. European Patent Application
EP2886659.4. Available from http://worldwide.espacenet.com/ publicationDetails/biblio? DB=worldwide.espacenet.com\&II=1\&ND= $3 \&$ adjacent $=$ true $\&$ locale $=$ en_EP $\& F T=D \&$ date $=20150625 \& C C=$ WO\&NR $=2015091979 A 2 \& K C=A 2$.

Weinhäusel A, Pichler R, Noehammer C (2009) Methylation Assay. European Patent Application EP 09450020.4. Available from: http:// worldwide.espacenet.com/publicationDetails/biblio?DB=worldwide. espacenet.com \&II $=4 \& \mathrm{ND}=3 \&$ adjacent $=$ true \&locale $=$ en_EP\&FT $=$ D\&date $=20111124 \& C C=$ US \&NR $=2011287968$ A $1 \&$ KC $=$ A 1 .

Weisenberger DJ, Siegmund KD, Campan M, Young J, Long TI, Faasse MA, Kang GH, Widschwendter M, Weener D, Buchanan D, Koh H, Simms L, Barker M, Leggett B, Levine J, Kim M, French AJ, Thibodeau SN, Jass J, Haile R, Laird PW (2006) CpG island methylator phenotype underlies sporadic microsatellite instability and is tightly associated with BRAF mutation in colorectal cancer. Nat Genet 38(7): 787-793.

Yagi K, Akagi K, Hayashi H, Nagae G, Tsuji S, Isagawa T, Midorikawa Y, Nishimura Y, Sakamoto H, Seto Y, Aburatani H, Kaneda A (2010) Three DNA methylation epigenotypes in human colorectal cancer. Clin Cancer Res 16(1): 21-33.

Yamauchi M, Morikawa T, Kuchiba A, Imamura Y, Qian ZR, Nishihara R, Liao X, Waldron L, Hoshida Y, Huttenhower C, Chan AT, Giovannucci E, Fuchs C, Ogino S (2012) Assessment of colorectal cancer molecular features along bowel subsites challenges the conception of distinct dichotomy of proximal versus distal colorectum. Gut 61(6): 847-854.

This work is published under the standard license to publish agreement. After 12 months the work will become freely available and the license terms will switch to a Creative Commons AttributionNonCommercial-Share Alike 4.0 Unported License.

Supplementary Information accompanies this paper on British Journal of Cancer website (http://www.nature.com/bjc) 